\title{
On Airy Solutions of the Second Painlevé Equation
}

\author{
By Peter A. Clarkson
}

In this paper we discuss Airy solutions of the second Painlevé equation and two related equations, the Painlevé XXXIV equation and the JimboMiwa-Okamoto $\sigma$ form of second Painlevé equation, are discussed. It is shown that solutions which depend only on the Airy function $\operatorname{Ai}(z)$ have a completely difference structure to those which involve a linear combination of the Airy functions $\operatorname{Ai}(z)$ and $\operatorname{Bi}(z)$.

\section{Dedicated to Mark Ablowitz on his 70th birthday}

\section{Introduction}

The six Painlevé equations $\left(\mathrm{P}_{\mathrm{I}}-\mathrm{P}_{\mathrm{VI}}\right)$ were first discovered by Painlevé, Gambier and their colleagues in an investigation of which second order ordinary differential equations of the form

$$
\frac{d^{2} q}{d z^{2}}=F\left(\frac{d q}{d z}, q, z\right),
$$

where $F$ is rational in $d q / d z$ and $q$ and analytic in $z$, have the property that their solutions have no movable branch points. They showed that there were fifty canonical equations of the form (1.1) with this property, now known as the Painlevé property. Further Painlevé, Gambier and their colleagues showed that of these fifty equations, forty-four can be reduced to linear equations, solved in terms of elliptic functions, or are reducible to one of six new nonlinear ordinary differential equations that define new transcendental functions, see Ince [1]. The Painlevé equations can be thought of as nonlinear analogues of the classical special functions $[2,3,4,5,6]$, and arise in a wide variety of applications, for example random matrices, cf. [7, 8].

Address for correspondence: School of Mathematics, Statistics \& Actuarial Science University of Kent, Canterbury, CT2 7NF, UK P.A.Clarkson@kent.ac.uk 
In this paper we are concerned with special solutions of the second Painlevé equation $\left(\mathrm{P}_{\mathrm{II}}\right)$

$$
\frac{d^{2} q}{d z^{2}}=2 q^{3}+z q+\alpha
$$

with $\alpha$ an arbitrary constant, and two related equations. These are thee Painlevé XXXIV equation $\left(\mathrm{P}_{34}\right)$

$$
\frac{d^{2} p}{d z^{2}}=\frac{1}{2 p}\left(\frac{d p}{d z}\right)^{2}+2 p^{2}-z p-\frac{\left(\alpha+\frac{1}{2}\right)^{2}}{2 p}
$$

is equivalent to equation XXXIV of Chapter 14 in [1], which is solvable in terms of $\mathrm{P}_{\mathrm{II}}(1.2)$, see $\S 2.1$, and the Jimbo-Miwa-Okamoto $\sigma$ form of $\mathrm{P}_{\mathrm{II}}\left(\mathrm{S}_{\mathrm{II}}\right)$

$$
\left(\frac{d^{2} \sigma}{d z^{2}}\right)^{2}+4\left(\frac{d \sigma}{d z}\right)^{3}+2 \frac{d \sigma}{d z}\left(z \frac{d \sigma}{d z}-\sigma\right)=\frac{1}{4}\left(\alpha+\frac{1}{2}\right)^{2}
$$

which is satisfied by the Hamiltonian associated with $\mathrm{P}_{\mathrm{II}}$ (1.2), see $\S 2.1$. Equation (1.4) is equation SD-I.d in the classification of second-order, second-degree equations which have the Painlevé property by Cosgrove and Scoufis [9], an equation first derived by Chazy [10]. Frequently in applications it is the associated second-order, second-degree equation such as (1.4) which arises rather than the Painlevé equation.

It is well-known that $\mathrm{P}_{\mathrm{II}}$ has special solutions depending on one parameter that are expressed in terms of the Airy functions $\operatorname{Ai}(z)$ and $\operatorname{Bi}(z)$. In this paper we study the Airy solutions for $\mathrm{P}_{\mathrm{II}}(1.2), \mathrm{P}_{34}(1.3)$ and $\mathrm{S}_{\mathrm{II}}$ (1.4), see $\S 3$. In particular it is shown that the solutions which depend only on $\operatorname{Ai}(z)$ have a completely different asymptotic behaviour as $z \rightarrow-\infty$ in comparison to those which involve a linear combination of $\operatorname{Ai}(z)$ and $\operatorname{Bi}(z)$. Further it is shown that there are families of Airy solutions of $\mathrm{P}_{34}$ (1.3) and $\mathrm{S}_{\mathrm{II}}(1.4)$ which have no poles on the real axis.

\section{Some properties of the second Painlevé equation}

\subsection{Hamiltonian structure}

Each of the Painlevé equations $\mathrm{P}_{\mathrm{I}}-\mathrm{P}_{\mathrm{VI}}$ can be written as a Hamiltonian system

$$
\frac{d q}{d z}=\frac{\partial \mathcal{H}_{\mathrm{J}}}{\partial p}, \quad \frac{d p}{d z}=-\frac{\partial \mathcal{H}_{\mathrm{J}}}{\partial q},
$$

for a suitable Hamiltonian function $\mathcal{H}_{\mathrm{J}}(q, p, z)[11,12,13,14]$. The function $\sigma(z) \equiv \mathcal{H}_{\mathrm{J}}(q, p, z)$ satisfies a second-order, second-degree ordinary 
differential equation, whose solution is expressible in terms of the solution of the associated Painlevé equation $[11,12,13,14]$.

The Hamiltonian associated with $\mathrm{P}_{\mathrm{II}}(1.2)$ is

$$
\mathcal{H}_{\mathrm{II}}(q, p, z ; \alpha)=\frac{1}{2} p^{2}-\left(q^{2}+\frac{1}{2} z\right) p-\left(\alpha+\frac{1}{2}\right) q
$$

and so

$$
\frac{d q}{d z}=p-q^{2}-\frac{1}{2} z, \quad \frac{d p}{d z}=2 q p+\alpha+\frac{1}{2}
$$

(Jimbo and Miwa [11], Okamoto [14]). Eliminating $p$ in (2.3) then $q$ satisfies $\mathrm{P}_{\mathrm{II}}(1.2)$ whilst eliminating $q$ yields (1.3). Further if $q$ satisfies $\mathrm{P}_{\mathrm{II}}$ (1.2) then $p=q^{\prime}+q^{2}+\frac{1}{2} z$ satisfies (1.3). Conversely if $p$ satisfies (1.3) then $q=\left(p^{\prime}-\alpha-\frac{1}{2}\right) /(2 p)$ satisfies $\mathrm{P}_{\mathrm{II}}(1.2)$. Thus there is a one-to-one correspondence between solutions of $\mathrm{P}_{\mathrm{II}}$ (1.2) and those of $\mathrm{P}_{34}$ (1.3).

An important property of the Hamiltonian (2.2), which is very useful in applications, is that it satisfies a second-order, second-degree ordinary differential equation, as discussed in the following theorem.

THEOREM 2.1. Consider the function $\sigma(z ; \alpha)=\mathcal{H}_{\mathrm{II}}(q, p, z ; \alpha)$ defined by (2.2), where $q$ and $p$ satisfy the system (2.3), then then $\sigma \sigma(z ; \alpha)$ satisfies (1.4). Conversely if $\sigma(z ; \alpha)$ is a solution of (1.4), then

$$
q(z ; \alpha)=\frac{4 \sigma^{\prime \prime}(z ; \alpha)+2 \alpha+1}{8 \sigma^{\prime}(z ; \alpha)}, \quad p(z ; \alpha)=-2 \sigma^{\prime}(z ; \alpha),
$$

with ${ }^{\prime} \equiv d / d z$, are solutions of (1.2) and (1.3), respectively.

Proof: See Jimbo and Miwa [11] and Okamoto [12, 13, 14].

\subsection{Bäcklund transformations}

The Painlevé equations $\mathrm{P}_{\mathrm{II}}-\mathrm{P}_{\mathrm{VI}}$ possess Bäcklund transformations which relate one solution to another solution either of the same equation, with different values of the parameters, or another equation (see $[2,4,15]$ and the references therein). An important application of the Bäcklund transformations is that they generate hierarchies of classical solutions of the Painlevé equations, which are discussed in $\S 3$.

The Bäcklund transformations for $\mathrm{P}_{\mathrm{II}}$ (1.2) are given in the following theorem.

TheOREm 2.2. Let $q \equiv q(z ; \alpha)$ is a solution of $\mathrm{P}_{\mathrm{II}}$ (1.2), then the transformations

$$
\begin{aligned}
\mathcal{S}: & q(z ;-\alpha)=-q, \\
\mathcal{T}_{ \pm}: & q(z ; \alpha \pm 1)=-q-\frac{2 \alpha \pm 1}{2 q^{2} \pm 2 q^{\prime}+z}
\end{aligned}
$$


give solutions of $\mathrm{P}_{\mathrm{II}}$, provided that $\alpha \neq \mp \frac{1}{2}$ in (2.6).

Proof: See Gambier [16].

The solutions $q_{\alpha}=q(z ; \alpha), q_{\alpha \pm 1}=q(z ; \alpha \pm 1)$ also satisfy the nonlinear recurrence relation

$$
\frac{2 \alpha+1}{q_{\alpha+1}+q_{\alpha}}+\frac{2 \alpha-1}{q_{\alpha}+q_{\alpha-1}}+4 q_{\alpha}^{2}+2 z=0,
$$

a difference equation which is known as an alternative form of discrete $\mathrm{P}_{\mathrm{I}}$ (alt- $\mathrm{dP}_{\mathrm{I}}$ ) [17]. The difference equation (2.7) is obtained by eliminating $q^{\prime}$ between the transformations $\mathcal{T}_{ \pm}$given by (2.6). Note that for $\mathrm{P}_{\text {II }}(1.2)$, the independent variable $z$ varies and the parameter $\alpha$ is fixed, whilst for the discrete equation $(2.7), z$ is a fixed parameter and $\alpha$ varies.

\section{Special function solutions}

The Painlevé equations $\mathrm{P}_{\mathrm{II}}-\mathrm{P}_{\mathrm{VI}}$ possess hierarchies of solutions expressible in terms of classical special functions, for special values of the parameters through an associated Riccati equation,

$$
\frac{d q}{d z}=f_{2}(z) q^{2}+f_{1}(z) q+f_{0}(z)
$$

where $f_{2}(z), f_{1}(z)$ and $f_{0}(z)$ are rational functions. Hierarchies of solutions, which are often referred to as "one-parameter solutions" (since they have one arbitrary constant), are generated from "seed solutions" derived from the Riccati equation using the Bäcklund transformations given in $\S 2.2$. Furthermore, as for the rational solutions, these special function solutions are often expressed in the form of determinants.

Solutions of $\mathrm{P}_{\mathrm{II}}-\mathrm{P}_{\mathrm{VI}}$ are expressed in terms of special functions as follows (see $[2,4,18]$, and the references therein): for $\mathrm{P}_{\mathrm{II}}$ in terms of Airy functions $\operatorname{Ai}(z), \operatorname{Bi}(z)$; for $\mathrm{P}_{\text {III }}$ in terms of Bessel functions $J_{\nu}(z), Y_{\nu}(z)$; for $\mathrm{P}_{\mathrm{IV}}$ in terms of parabolic cylinder functions functions $D_{\nu}(z)$; for $\mathrm{P}_{\mathrm{V}}$ in terms of confluent hypergeometric functions ${ }_{1} F_{1}(a ; c ; z)$, equivalently Kummer functions $M(a, b, z), U(a, b, z)$ or Whittaker functions $M_{\kappa, \mu}(z)$, $q_{\kappa, \mu}(z)$; and for $\mathrm{P}_{\mathrm{VI}}$ in terms of hypergeometric functions ${ }_{2} F_{1}(a, b ; c ; z)$. Some classical orthogonal polynomials arise as particular cases of these special function solutions and thus yield rational solutions of the associated Painlevé equations: for $\mathrm{P}_{\mathrm{III}}$ and $\mathrm{P}_{\mathrm{V}}$ in terms of associated Laguerre polynomials $L_{k}^{(m)}(z)$; for $\mathrm{P}_{\mathrm{IV}}$ in terms of Hermite polynomials $H_{n}(z)$; and for $\mathrm{P}_{\mathrm{VI}}$ in terms of Jacobi polynomials $P_{n}^{(\alpha, \beta)}(z)$. 


\subsection{Second Painlevé equation}

We note that $\mathrm{P}_{\mathrm{II}}(1.2)$ can be written as

$$
\varepsilon \frac{d}{d z}\left(\varepsilon \frac{d q}{d z}-q^{2}-\frac{1}{2} z\right)+2 q\left(\varepsilon \frac{d q}{d z}-q^{2}-\frac{1}{2} z\right)=\alpha-\frac{1}{2} \varepsilon,
$$

with $\varepsilon^{2}=1$. Hence if $\alpha=\frac{1}{2} \varepsilon$, then special solutions of $\mathrm{P}_{\mathrm{II}}$ can be obtained in terms of solutions of the Riccati equation

$$
\varepsilon \frac{d q}{d z}=q^{2}+\frac{1}{2} z
$$

Any solution of this equation is also a solution of $\mathrm{P}_{\mathrm{II}}(1.2)$, provided that $\alpha=\frac{1}{2} \varepsilon$. Linearising the Riccati equation (3.2) by setting $q=-\varepsilon \varphi^{\prime} / \varphi$ yields

$$
\frac{d^{2} \varphi}{d z^{2}}+\frac{1}{2} z \varphi=0
$$

which is equivalent to the Airy equation and has general solution

$$
\varphi(z ; \vartheta)=\cos (\vartheta) \operatorname{Ai}(t)+\sin (\vartheta) \operatorname{Bi}(t), \quad t=-2^{-1 / 3} z,
$$

with $\operatorname{Ai}(t)$ and $\operatorname{Bi}(t)$ the Airy functions and $\vartheta$ an arbitrary constant. The Airy solutions of $\mathrm{P}_{\mathrm{II}}$ are classified in the following theorem due to Gambier [16].

Theorem 3.1. The second Painlevé equation (1.2) has a oneparameter family of solutions expressible in terms of Airy functions given by (3.4) if and only if $\alpha=n-\frac{1}{2}$, with $n \in \mathbb{Z}$.

The simplest Airy solutions of $\mathrm{P}_{\mathrm{II}}(1.2)$, which arise when $\alpha= \pm \frac{1}{2}$, are

$$
q(z)=\mp \frac{d}{d z} \ln \varphi(z ; \vartheta)
$$

with $\varphi(z ; \vartheta)$ given by (3.4). Then using the Bäcklund transformations (2.6), a hierarchy of Airy solutions for $\alpha=n+\frac{1}{2}, n \in \mathbb{Z}$, can be generated. The Airy solutions can also be expressed in terms of determinants, as described in the following theorem.

THEOREM 3.2. Let $\tau_{n}(z ; \vartheta)$ be the $n \times n$ determinant

$$
\tau_{n}(z ; \vartheta)=\left[\frac{d^{j+k}}{d z^{j+k}} \varphi(z ; \vartheta)\right]_{j, k=0}^{n-1}, \quad n \geq 1,
$$

with $\varphi(z ; \vartheta)$ given by (3.4) and $\tau_{0}(z ; \vartheta)=1$, then

$$
q_{n}(z ; \vartheta)=\frac{d}{d z} \ln \frac{\tau_{n-1}(z ; \vartheta)}{\tau_{n}(z ; \vartheta)}, \quad n \geq 1
$$




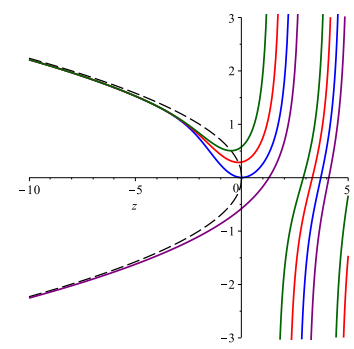

$n=1$

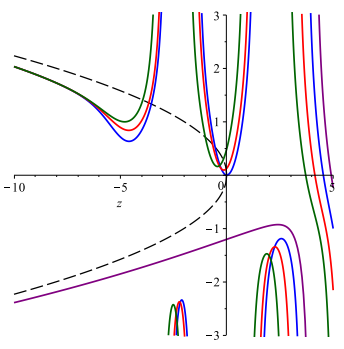

$n=4$

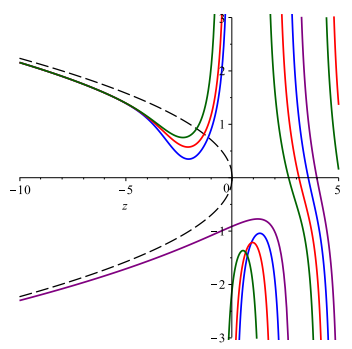

$n=2$

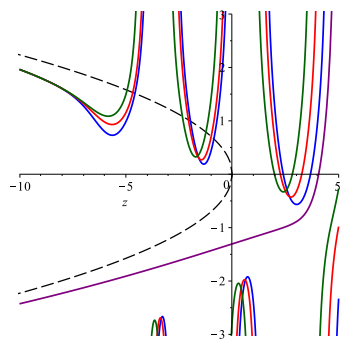

$n=5$

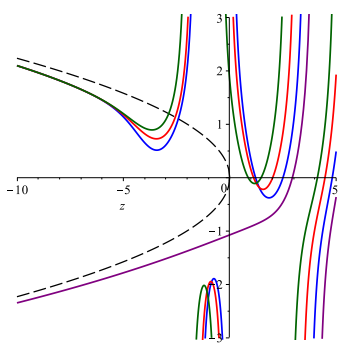

$n=3$

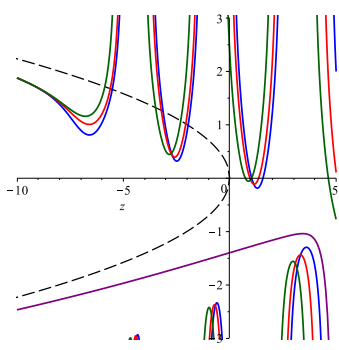

$n=6$

Figure 3.1. Plots of $q_{n}(z ; \vartheta)(3.7)$ for $\vartheta=0$ [purple], $\vartheta=\frac{1}{6} \pi$ [blue], $\vartheta=\frac{1}{3} \pi$ [red], $\vartheta=\frac{1}{2} \pi$ [green]; the dashed line is the parabola $2 q^{2}+z=0$.

\begin{tabular}{|c|c|}
\hline$n$ & $q_{n}(z ; \vartheta)$ \\
\hline 1 & $\Phi$ \\
3 & $-\Phi-\frac{1}{2 \Phi^{2}+z}$ \\
4 & $\frac{2 z \Phi^{2}-\Phi+z^{2}}{4 \Phi^{3}+2 z \Phi+1}-\frac{2 z \Phi^{2}-\Phi+z^{2}}{z\left(8 z \Phi^{4}-16 \Phi^{3}+8 z^{2} \Phi^{2}-8 z \Phi+2 z^{3}-3\right)}-\frac{3}{z}$ \\
\hline
\end{tabular}

Table 3.1

The Airy solutions $q_{n}(z ; \vartheta)$ of $\mathrm{P}_{\mathrm{II}}(1.2)$, see (3.7), where $\Phi=-\varphi^{\prime}(z ; \vartheta) / \varphi(z ; \vartheta)$, with $\varphi(z ; \vartheta)$ given by $(3.4)$. 
satisfies $\mathrm{P}_{\mathrm{II}}$ (1.2) with $\alpha=n-\frac{1}{2}$.

Proof: See Flaschka and Newell [19], Okamoto [14]; also [20].

We remark that the determinant

$$
\Delta_{n}(t)=\operatorname{det}\left[\frac{d^{j+k}}{d t^{j+k}} \operatorname{Ai}(t)\right]_{j, k=0}^{n-1}
$$

which is equivalent to $\tau_{n}(z ; 0)$ given by (3.6), arises in random matrix theory, in connection with the Gaussian Unitary Ensemble (GUE) in the soft-edge scaling limit, see e.g. [20, p. 393].

If we set $\Phi(z ; \vartheta) \equiv-\varphi^{\prime}(z ; \vartheta) / \varphi(z ; \vartheta)$, with $\varphi(z)$ given by $(3.4)$, then the first few solutions in the Airy function solution hierarchy for $\mathrm{P}_{\text {II }}(1.2)$ are given in Table 3.1. We note that $\Phi(z ; \vartheta)$ satisfies the Riccati equation (3.2) with $\varepsilon=1$.

Plots of the solutions $q_{n}(z ; \vartheta)(3.7)$ for various $\vartheta$ are given in Figure 3.1. These plots show that the asymptotic behaviour as $z \rightarrow-\infty$ of the solutions is completely different in the case when $\vartheta=0$ compared to the case when $\vartheta \neq 0$, see Theorem 3.3. Fornberg and Weideman [21, Figure 3 on p. 991] plot the locations of the poles for the solution $q\left(z ; \frac{5}{2}\right)$ of $\mathrm{P}_{\mathrm{II}}$ (1.2) for various choices of $\vartheta$, which show that the pole structure of the solutions is significantly different in the case when $\vartheta=0$ compared to the case when $\vartheta \neq 0$.

When $\vartheta=0$ the solution $q_{n}(z ; 0)$ involves only on the Airy function $\operatorname{Ai}(t)$, with $t=-2^{-1 / 3} z$, whereas the solution $q_{n}(z ; \vartheta)$, with $\vartheta \neq 0$, involves the Airy function $\operatorname{Bi}(t)$. The known asymptotics of $\operatorname{Ai}(t)$ and $\operatorname{Bi}(t)$ as $t \rightarrow \infty$ are

$$
\begin{aligned}
& \operatorname{Ai}(t)=\frac{1}{2} \pi^{-1 / 2} t^{-1 / 4} \exp \left(-\frac{2}{3} t^{3 / 2}\right)\left\{1+\mathcal{O}\left(t^{-3 / 2}\right)\right\}, \\
& \operatorname{Bi}(t)=\pi^{-1 / 2} t^{-1 / 4} \exp \left(\frac{2}{3} t^{3 / 2}\right)\left\{1+\mathcal{O}\left(t^{-3 / 2}\right)\right\},
\end{aligned}
$$

cf. [22, §9.7(ii)]. Consequently the asymptotic behaviour of $q_{n}(z ; \vartheta)$ as $z \rightarrow-\infty$ critically depends on whether it involves $\operatorname{Bi}(t)$.

The asymptotic behaviour of the Airy solutions $q_{n}(z ; \vartheta)$ as $z \rightarrow-\infty$ is given in the following theorem.

THEOREM 3.3. Let $q_{n}(z ; \vartheta)$ be defined by (3.7), then as $z \rightarrow-\infty$, $q_{n}(z ; \vartheta)= \begin{cases}-\frac{(-z)^{1 / 2}}{\sqrt{2}}+\frac{2 n-1}{4 z}+\frac{12 n^{2}-12 n+5}{16 \sqrt{2}(-z)^{5 / 2}}+\mathcal{O}\left(z^{-4}\right), & \text { if } \vartheta=0, \\ \frac{(-z)^{1 / 2}}{\sqrt{2}}+\frac{2 n-1}{4 z}-\frac{12 n^{2}-12 n+5}{16 \sqrt{2}(-z)^{5 / 2}}+\mathcal{O}\left(z^{-4}\right), & \text { if } \vartheta \neq 0 .\end{cases}$

Proof: These are proved using the asymptotics (3.8) of $\mathrm{Ai}(t)$ and $\mathrm{Bi}(t)$ as $t \rightarrow \infty$; for details, see Clarkson, Loureiro, and Van Assche [23]. 


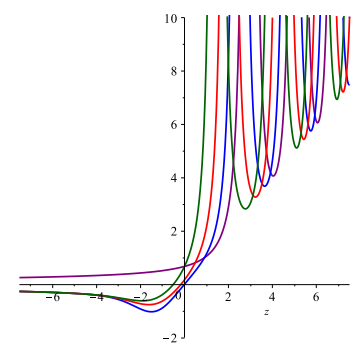

$n=1$

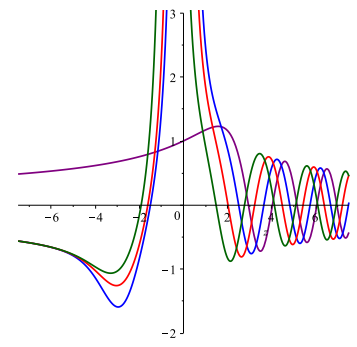

$n=2$

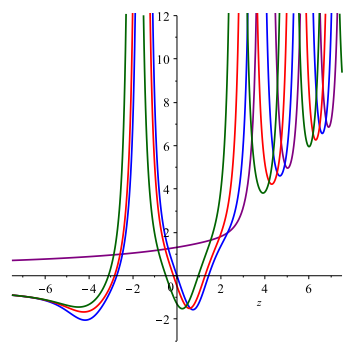

$n=3$

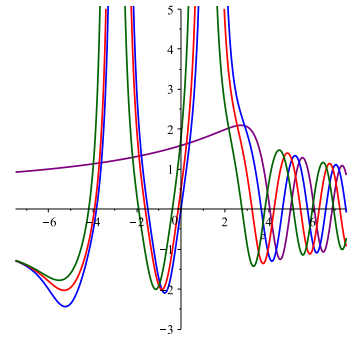

$n=4$

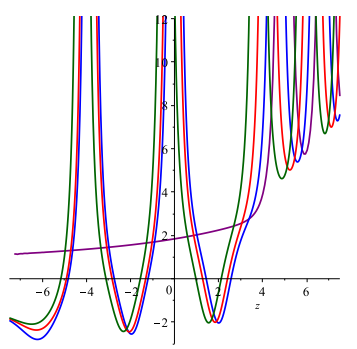

$n=5$

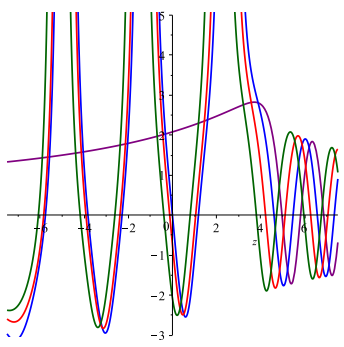

$n=6$

Figure 3.2. Plots of $p_{n}(z ; \vartheta) / n$, with $p_{n}(z ; \vartheta)$ given by (3.9), for $\vartheta=0$ [purple], $\vartheta=\frac{1}{6} \pi$ [blue], $\vartheta=\frac{1}{3} \pi$ [red], $\vartheta=\frac{1}{2} \pi$ [green].

We remark that Its, Kuijlaars, and Östensson [24] noted that $p_{1}(z ; 0)$ is special among all solutions $p_{1}(z ; \vartheta)$ in its behavior as $z \rightarrow-\infty$.

The plots in Figure 3.1 suggest the following conjecture (see also [23]).

Conjecture 3.1: If $q_{n}(z ; 0)$ is defined by (3.7), then for $z<0$ and $n \geq 1, q_{n}(z ; 0)$ is a monotonically decreasing function and

$$
q_{n+1}(z ; 0)<q_{n}(z ; 0) .
$$

\subsection{Painlevé XXXIV equation and Jimbo-Miwa-Okamoto $\sigma$ equation}

Due to the one-to-one correspondence between solutions of $\mathrm{P}_{\mathrm{II}}(1.2)$ and those of $\mathrm{P}_{34}$ (1.3) and $\mathrm{S}_{\text {II }}$ (1.4), as discussed in $\S 2.1$, we have the following result.

THEOREM 3.4. Equations (1.3) and (1.4) possess one-parameter family of solutions expressible in terms of Airy functions given by (3.4) if and only if $\alpha=n-\frac{1}{2}$, with $n \in \mathbb{Z}$.

As for the Airy solutions of $\mathrm{P}_{\mathrm{II}}$ (1.2), the Airy solutions of $\mathrm{P}_{34}$ (1.3) and $\mathrm{S}_{\mathrm{II}}$ (1.4) can be expressed in terms of the determinant (3.6). 


\begin{tabular}{|c|c|}
\hline$n$ & $p_{n}(z ; \vartheta)$ \\
\hline 1 & $2 \Phi^{2}+z$ \\
2 & $\frac{4 \Phi}{2 \Phi^{2}+z}+\frac{2}{\left(2 \Phi^{2}+z\right)^{2}}$ \\
3 & $z-\frac{8 \Phi^{2}-2 z^{2} \Phi+6 z}{4 \Phi^{3}+2 z \Phi+1}+\frac{2\left(2 z^{2}+1\right) \Phi^{2}-2 z^{2} \Phi+2 z\left(z^{3}-1\right)}{\left(4 \Phi^{3}+2 z \Phi+1\right)^{2}}$ \\
\hline
\end{tabular}

Table 3.2

The Airy solutions $p_{n}(z ; \vartheta)$ of $\mathrm{P}_{34}(1.3)$ given by (3.9).

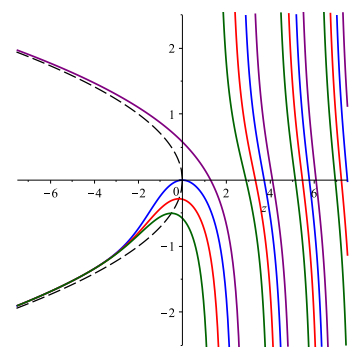

$n=1$

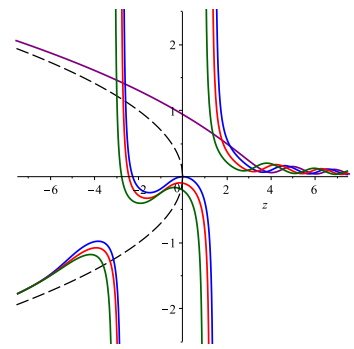

$n=4$

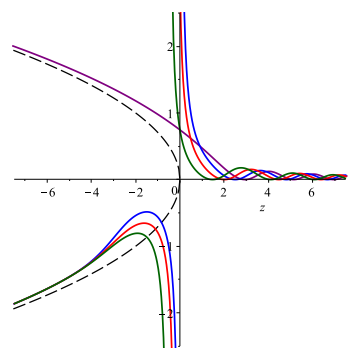

$n=2$

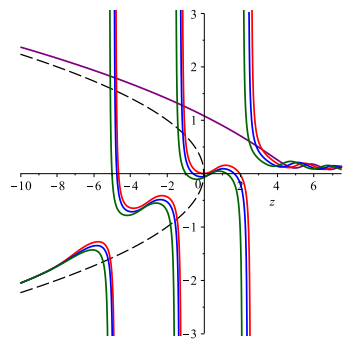

$n=6$

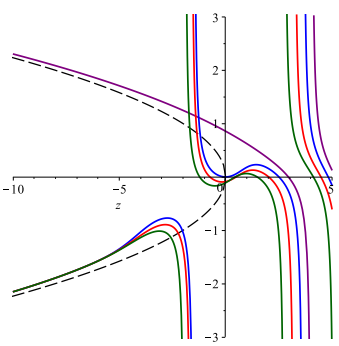

$n=3$

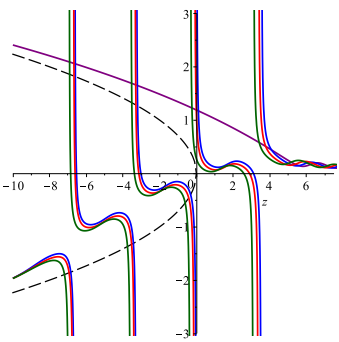

$n=8$

Figure 3.3. Plots of $\sigma_{n}(z ; \vartheta) / n$, with $\sigma_{n}(z ; \vartheta)$ given by $(3.10)$, for $\vartheta=0$ [purple], $\vartheta=\frac{1}{6} \pi$ [blue], $\vartheta=\frac{1}{3} \pi$ [red], $\vartheta=\frac{1}{2} \pi$ [green]; the dashed line is the parabola $2 \sigma^{2}+z=0$. 


\begin{tabular}{|c|c|}
\hline$n$ & $\vartheta_{n}(z ; \vartheta)$ \\
\hline 1 & $-\Phi$ \\
2 & $\frac{1}{2 \Phi^{2}+z}$ \\
3 & $\frac{2 z \Phi^{2}-\Phi+z^{2}}{4 \Phi^{3}+2 z \Phi+1}$ \\
4 & $\frac{48 \Phi^{3}-8 z^{2} \Phi^{2}+28 z \Phi-4 z^{3}+9}{z\left(8 z \Phi^{4}-16 \Phi^{3}+8 z^{2} \Phi^{2}-8 z \Phi+2 z^{3}-3\right)}+\frac{3}{z}$ \\
\hline
\end{tabular}

Table 3.3

The Airy solutions $\vartheta_{n}(z ; \vartheta)(3.10)$.

Theorem 3.5. Let $\tau_{n}(z ; \vartheta)$ be the determinant (3.6) for $n \geq 1$, with $\varphi(z ; \vartheta)$ given by $(3.4)$ and $\tau_{0}(z ; \vartheta)=1$, then

$$
\begin{aligned}
& p_{n}(z ; \vartheta)=-2 \frac{d^{2}}{d z^{2}} \ln \tau_{n}(z ; \vartheta), \\
& \sigma_{n}(z ; \vartheta)=\frac{d}{d z} \ln \tau_{n}(z ; \vartheta),
\end{aligned}
$$

satisfy $\mathrm{P}_{34}$ (1.3) and $\mathrm{S}_{\mathrm{II}}$ (1.4) with $\alpha=n-\frac{1}{2}$, respectively.

Comparing (3.7) and (3.10), we see that

$$
q_{n}(z ; \vartheta)=\sigma_{n-1}(z ; \vartheta)-\sigma_{n}(z ; \vartheta) .
$$

The first few Airy solutions of $\mathrm{P}_{34}$ (1.3) and $\mathrm{S}_{\mathrm{II}}$ (1.4) are given in Tables 3.2 and 3.3 , respectively. Plots of these solutions are given in Figures 3.2 and 3.3, respectively. As was the case for $\mathrm{P}_{\mathrm{II}}$, these plots show that the asymptotic behaviour as $z \rightarrow-\infty$ of the Airy solutions is completely different when $\vartheta=0$ compared to the case when $\vartheta \neq 0$. We see that the solutions $p_{2 n}(z ; 0)$ and $\sigma_{2 n}(z ; 0)$, for $n \in \mathbb{Z}$, are special in that they have no poles on the real axis, see also Figures 3.4 and 3.5. Further these solutions have oscillatory behaviour with algebraic decay as $z \rightarrow \infty$ as given in Theorem 3.7, see also Figures 3.6 and 3.7.

The asymptotic behaviour of the Airy solutions $p_{n}(z ; \vartheta)$ and $\sigma_{n}(z ; \vartheta)$ as $z \rightarrow-\infty$ is given in the following theorem. 

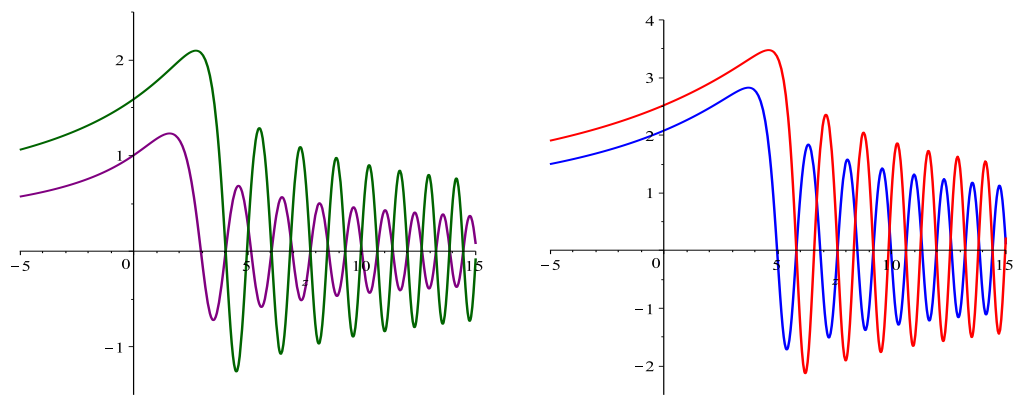

$$
n=2 \text { [purple], } n=4 \text { [green] }
$$

$$
n=6 \text { [blue], } n=8 \text { [red] }
$$

Figure 3.4. Plots of $p_{n}(z ; 0)(3.9)$, for $n=2,4,6,8$.

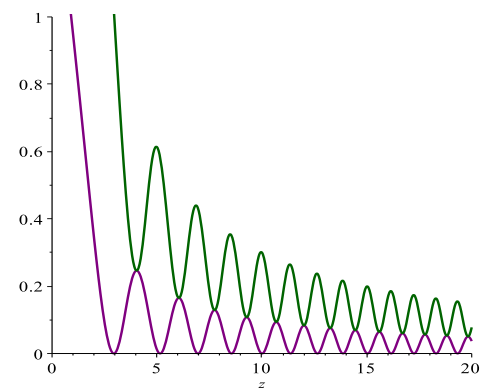

$n=2$ [purple], $n=4$ [green]

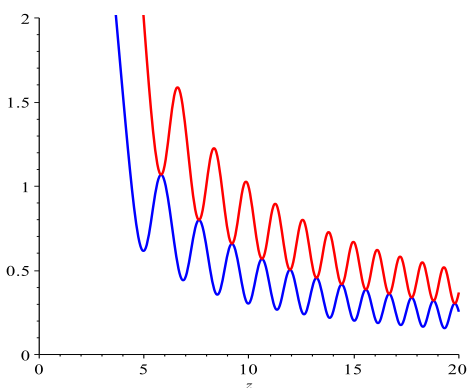

$n=6$ [blue], $n=8$ [red]

Figure 3.5. Plots of $\sigma_{n}(z ; 0)(3.10)$, for $n=2,4,6,8$.

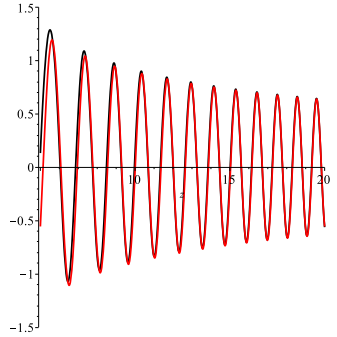

$n=4$

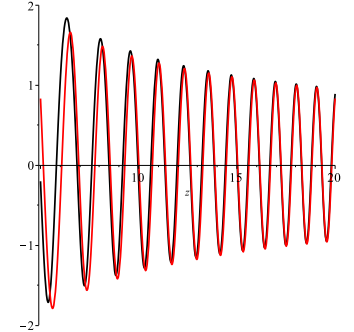

$n=6$

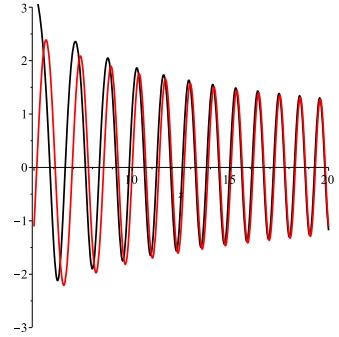

$n=8$

Figure 3.6. Plots of $p_{n}(z ; 0)(3.9)$ [blue] and the leading term in the asymptotic expansion (3.14) [red] for $n=4,6,8$. 


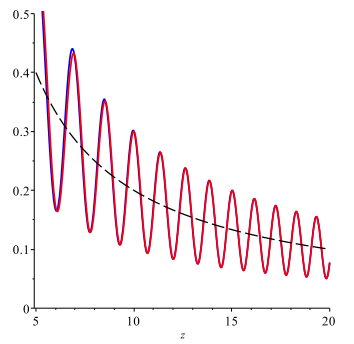

$n=4$

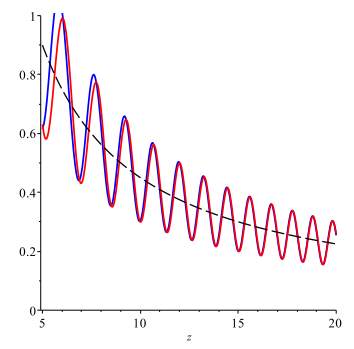

$n=6$

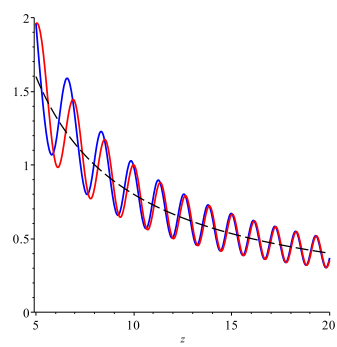

$n=8$

Figure 3.7. Plots of $\sigma_{n}(z ; 0)$ (3.10) [blue] and the leading term in the asymptotic expansion (3.15) [red] for $n=4,6,8$; the dashed line is $n^{2} /(8 z)$.

THEOREM 3.6. Let $p_{n}(z ; \vartheta)$ be defined by (3.9) and $\sigma_{n}(z ; \vartheta)$ by (3.10), then as $z \rightarrow-\infty$,

$p_{n}(z ; \vartheta)=\left\{\begin{array}{l}\frac{n}{\sqrt{2}(-z)^{1 / 2}}-\frac{n^{2}}{2 z^{2}}+\frac{5 n\left(4 n^{2}+1\right)}{16 \sqrt{2}(-z)^{7 / 2}}+\mathcal{O}\left(z^{-5}\right), \\ -\frac{n}{\sqrt{2}(-z)^{1 / 2}}-\frac{n^{2}}{2 z^{2}}-\frac{5 n\left(4 n^{2}+1\right)}{16 \sqrt{2}(-z)^{7 / 2}}+\mathcal{O}\left(z^{-5}\right), \quad \text { if } \quad \vartheta \neq 0,\end{array}\right.$

$\sigma_{n}(z ; \vartheta)= \begin{cases}\frac{n(-z)^{1 / 2}}{\sqrt{2}}-\frac{n^{2}}{4 z}-\frac{n\left(4 n^{2}+1\right)}{16 \sqrt{2}(-z)^{5 / 2}}+\mathcal{O}\left(z^{-4}\right), & \text { if } \quad \vartheta=0, \\ -\frac{n(-z)^{1 / 2}}{\sqrt{2}}-\frac{n^{2}}{4 z}+\frac{n\left(4 n^{2}+1\right)}{16 \sqrt{2}(-z)^{5 / 2}}+\mathcal{O}\left(z^{-4}\right), & \text { if } \quad \vartheta \neq 0 .\end{cases}$

Proof: The expansions (3.12) and (3.13) are easily derived using the expansion for $q_{n}(z ; \vartheta)$ given in Theorem 3.3 since if $q$ is solution of $\mathrm{P}_{\mathrm{II}}$ (1.2), then

$$
p=\frac{d q}{d z}+q^{2}+\frac{1}{2} z,
$$

is a solution of $\mathrm{P}_{34}$ (1.3), and

$$
\begin{aligned}
\sigma & =\frac{1}{2} p^{2}-\left(q^{2}+\frac{1}{2} z\right) p-\left(\alpha+\frac{1}{2}\right) q \\
& =\frac{1}{2}\left(\frac{d q}{d z}\right)^{2}-\frac{1}{2} q^{4}-\frac{1}{2} z q^{2}-\left(\alpha+\frac{1}{2}\right) q-\frac{1}{8} z^{2}
\end{aligned}
$$

is a solution of $\mathrm{S}_{\mathrm{II}}(1.4)$. 
The asymptotic behaviour of the solutions $p_{n}(z ; \vartheta)$ and $\sigma_{n}(z ; \vartheta)$ as $z \rightarrow \infty$, for $n \in 2 \mathbb{Z}$, is given in the following theorem.

TheOrem 3.7. Let $p_{n}(z ; \vartheta)$ be defined by (3.9) and $\sigma_{n}(z ; \vartheta)$ by (3.10) and $n \in 2 \mathbb{Z}$, then as $z \rightarrow \infty$,

$$
\begin{aligned}
& p_{n}(z ; 0)=\frac{1}{2} n \sqrt{2} z^{-1 / 2} \cos \left(\frac{4}{3} \sqrt{2} z^{3 / 2}-\frac{1}{2} n \pi\right)+o\left(z^{-1 / 2}\right), \\
& \sigma_{n}(z ; 0)=\frac{n}{8 z}\left\{n-2 \sin \left(\frac{4}{3} \sqrt{2} z^{3 / 2}-\frac{1}{2} n \pi\right)\right\}+o\left(z^{-1}\right) .
\end{aligned}
$$

Proof: Using the known asymptotic expansion of the Airy function as $t \rightarrow-\infty$, i.e.

$$
\operatorname{Ai}(t)=\pi^{-1 / 2}(-t)^{-1 / 4} \sin \left\{\frac{2}{3}(-t)^{3 / 2}+\frac{1}{4} \pi\right\}+o\left((-t)^{-1 / 4}\right)
$$

$[22, \S 9.7(\mathrm{ii})]$ it is straightforward to verify the asymptotic expansions (3.14) and (3.15) for small values of $n$.

Plots of $p_{n}(z ; 0)(3.9)$ and the leading term in the asymptotic expansion (3.14) for $n=4,6,8$ are given in Figure 3.6. Analogous plots of $\sigma_{n}(z ; 0)$ (3.10) and the leading term in the asymptotic expansion (3.15) for $n=$ 4, 6, 8 are given in Figure 3.7.

Remark 3.1. Its, Kuijlaars, and Östensson [24] discussed solution of the equation

$$
u_{\beta} \frac{d^{2} u_{\beta}}{d t^{2}}=\frac{1}{2}\left(\frac{d u_{\beta}}{d t}\right)^{2}+4 u_{\beta}^{3}+2 t u_{\beta}^{2}-2 \beta^{2},
$$

where $\beta$ is a constant, which is equivalent to $\mathrm{P}_{34}$ (1.3) through the transformation $p(z)=2^{1 / 2} u_{\beta}(t)$, with $t=-2^{-1 / 3} z$, and $\beta=\frac{1}{2} \alpha+\frac{1}{4}$ in their study of the double scaling limit of unitary random matrix ensembles of the form $Z_{n, N}^{-1}|\operatorname{det} M|^{2 \beta} \exp \{-N \operatorname{Tr} V(M)\} d M$, with $\beta>-\frac{1}{2}$ and $V$ real analytic. In particular,

$$
u_{1}(t)=-\frac{d^{2}}{d t^{2}} \mathcal{W}\left(\operatorname{Ai}(t), \mathrm{Ai}^{\prime}(t)\right),
$$

with $\mathcal{W}\left(\varphi_{1}, \varphi_{2}\right)$ the Wronskian, which is equivalent to the solution $p_{2}(z ; 0)$ of $\mathrm{P}_{34}$ (1.3), and noted that on the positive real axis, $u_{1}(t)$ has an infinite number of zeros, which are the zeros of the Airy function $\operatorname{Ai}(t)$, and an infinite number of additional zeros that interlace with the zeros of $\mathrm{Ai}(t)$, see Figure 3.8.

Remark 3.2. Its, Kuijlaars, and Östensson [25, Theorem 1.2] prove that there are solutions $u_{\beta}(t)$ of (3.16) such that as $t \rightarrow \infty$

$$
\begin{array}{ll}
u_{\beta}(t)=\beta t^{-1 / 2}+\mathcal{O}\left(t^{-2}\right), & \text { as } t \rightarrow \infty . \\
u_{\beta}(t)=\beta(-t)^{-1 / 2} \cos \left\{\frac{4}{3}(-t)^{3 / 2}-\beta \pi\right\}+\mathcal{O}\left(t^{-2}\right), & \text { as } t \rightarrow-\infty .
\end{array}
$$




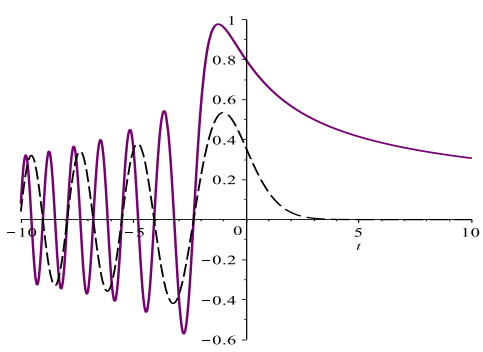

Figure 3.8. The solution $u_{1}(t)$ (3.17) of equation (3.16) [purple] and the Airy function $\operatorname{Ai}(t)$ [dashed line].

Letting $\beta=1$ in (3.18) and (3.19) shows that they are in agreement with (3.12) and (3.14), for $n=2$. Further Its, Kuijlaars, and Östensson conclude that solutions of (3.16) with asymptotic behaviour (3.18) are tronquée solutions, i.e. have no poles in a sector of the complex plane.

The plots in Figures 3.2 and 3.3 suggest the following conjecture, which is analogous to Conjecture 3.1 .

ConjeCture 3.2: If $p_{n}(z ; 0)$ is defined by $(3.9)$ and $\sigma_{n}(z ; 0)$ by $(3.10)$, then for $z<0$ and $n \geq 1, p_{n}(z ; 0)$ is a monotonically decreasing function and $\sigma_{n}(z ; 0)$ is a monotonically increasing function as $z$ decreases. Further, for $z<0$

$$
p_{n+1}(z ; 0)<p_{n}(z ; 0), \quad \sigma_{n}(z ; 0)<\sigma_{n+1}(z ; 0) .
$$

\section{Conclusion}

In this paper we have studied the Airy solutions of $\mathrm{P}_{\mathrm{II}}(1.2), \mathrm{P}_{34}$ (1.3), and $\mathrm{S}_{\text {II }}(1.2)$. These show that when the solutions depend only on the Airy function $\mathrm{Ai}(t)$, with $t=-2^{-1 / 3} z$, have a completely different asymptotic behaviour as $z \rightarrow-\infty$ compared to solutions which involve the Airy function $\operatorname{Bi}(t)$. The special solutions $q_{n}(z ; 0)$ and $p_{n}(z ; 0)$ of $\mathrm{P}_{\mathrm{II}}$ and $\mathrm{P}_{34}$ arise in recent study by Clarkson, Loureiro, and Van Assche [23] of the discrete system

$$
\begin{aligned}
& a_{n}+a_{n+1}=b_{n}^{2}-t, \\
& a_{n}\left(b_{n}+b_{n-1}\right)=n,
\end{aligned}
$$

which is equivalent to alt- $\mathrm{dP}_{\mathrm{I}}(2.7)$. The system (4.1) arises in the study of orthogonal polynomials with respect to an exponential cubic weight, see $[26,27,28]$. 


\section{Acknowledgments}

I would like to thank Mark Ablowitz, Andrew Bassom, Andrew Hone, Alexander Its, Kerstin Jordaan, Nalini Joshi, Ana Loureiro, Elizabeth Mansfield, Marta Mazzocco, Bryce McLeod, and Walter Van Assche for their helpful comments and illuminating discussions.

\section{References}

1. E.L. IncE, Ordinary Differential Equations, Dover, New York, 1956.

2. P.A. Clarkson, Painlevé equations - non-linear special functions, in Orthogonal Polynomials and Special Functions: Computation and Application, F. Marcellàn and W. Van Assche (Editors), Lect. Notes Math., vol. 1883, pp. 331-411, Springer-Verlag, Berlin, 2006.

3. A.S. Fokas, A.R. Its, A.A. Kapaev, and V.Yu. Novokshenov, Painlevé Transcendents: The Riemann-Hilbert approach, Math. Surv. Mono., vol. 128, American Mathematical Society, Providence, RI, 2006.

4. V.I. Gromak, I. Laine, and S. Shimomura, Painlevé Differential Equations in the Complex Plane, Studies in Math., vol. 28, de Gruyter, Berlin, New York, 2002.

5. K. Iwasaki, H. Kimura, S. Shimomura, and M. Yoshida, From Gauss to Painlevé: a Modern Theory of Special Functions, Aspects of Mathematics E, vol. 16, Viewag, Braunschweig, Germany, 1991.

6. H. Umemura, Painlevé equations and classical functions, Sugaku Expositions, 11:77-100 (1998).

7. P.J. Forrester, Log-gases and random matrices, London Math. Soc. Mono. Series, vol. 34, Princeton University Press, Princeton, NJ, 2010.

8. V.Al. Osipov and E. KAnziePer, Correlations of RMT characteristic polynomials and integrability: Hermitean matrices, Annals of Physics, 325:2251-2306 (2010).

9. C.M. Cosgrove and G. Scoufis, Painlevé classification of a class of differential equations of the second order and second-degree, Stud. Appl. Math., 88:25-87 (1993).

10. J. Chazy, Sur les équations différentielles du troisième ordre et d'ordre supérieur dont l'intégrale générale a ses points critiques fixes, Acta Math., 34:317-385 (1911).

11. M. Jiмво and T. MiwA, Monodromy preserving deformations of linear ordinary differential equations with rational coefficients. II, Physica, D2:407-448 (1981).

12. K. Окамото, Polynomial Hamiltonians associated with Painlevé equations. I, Proc. Japan Acad. Ser. A Math. Sci., 56:264-268 (1980).

13. K. Окамото, Polynomial Hamiltonians associated with Painlevé equations. II, Proc. Japan Acad. Ser. A Math. Sci., 56:367-371 (1980).

14. K. Окамото, Studies on the Painlevé equations III. Second and fourth Painlevé equations, $\mathrm{P}_{\mathrm{II}}$ and $\mathrm{P}_{\mathrm{IV}}$, Math. Ann., 275:221-255 (1986).

15. A.S. FokAS and M.J. ABlowitz, On a unified approach to transformations and elementary solutions of Painlevé equations, J. Math. Phys., 23:2033-2042 (1982).

16. B. Gambier, Sur les équations différentielles du second ordre et du premeir degre dont l'intégrale générale est à points critiques fixés, Acta Math., 33:1-55 (1909).

17. A.S. Fokas, B. Grammaticos, and A. Ramani, From continuous to discrete Painlevé equations, J. Math. Anal. Appl., 180:342-360 (1993). 
18. T. Masuda, Classical transcendental solutions of the Painlevé equations and their degeneration, Tohoku Math. J. (2), 56:467-490 (2004).

19. H. Flaschka and A.C. Newell, Monodromy- and spectrum preserving deformations. I, Commun. Math. Phys., 76:65-116 (1980).

20. P.J. Forrester and N.S. Witte, Application of the $\tau$-function theory of Painlevé equations to random matrices: PIV, PII and the GUE, Commun. Math. Phys., 219:357-398 (2001).

21. B. Fornberg and J.A.C. Weideman, A computational exploration of the second Painlevé equation, Found. Comput. Math., 14:985-1016 (2014).

22. F.W.J. Olver, D.W. Lozier, R.F. Boisvert, and C.W. Clark (Editors), NIST Handbook of Mathematical Functions, C.U.P., Cambridge, 2010.

23. P.A. Clarkson, A.F. Loureiro, and W. Van Assche, Unique positive solution for an alternative discrete Painlevé I equation, arXiv:1508.04916 [nlin.SI] (2015).

24. A.R. Its, A.B.J. KuiJlaArs, and J. Östensson, Critical edge behavior in unitary random matrix ensembles and the thirty fourth Painlevé transcendent, Int. Math. Res. Not., 2008:rnn017 (2008).

25. A.R. Its, A.B.J. Kuijlaars, and J. Östensson, Asymptotics for a special solution of the thirty fourth Painlevé equation, Nonlinearity, 22:1523-1558 (2009).

26. P.M. Bleher and A. DeAÑo, Topological expansion in the cubic random matrix model, Int. Math. Res. Not. IMRN, :no. 12, pp. 2699-2755 (2013).

27. G. Filipuk, W. Van Assche, and L. Zhang, Multiple orthogonal polynomials with an exponential cubic weight, J. Approx. Theory, 193:1-25 (2015).

28. A. Magnus, Painlevé-type differential equations for the recurrence coefficients of semi-classical orthogonal polynomials, J. Comput. Appl. Math., 57:215-237 (1995).

School of Mathematics, Statistics \& Actuarial Science University of Kent, Canterbury, CT2 7NF, UK 